\title{
OntoTrans: An Ontology on Transparency
}

\author{
Arthur M. Pereira ${ }^{1}$, Claudia Cappelli², Fernanda A. Baião ${ }^{3}$, Vanessa T. Nunes ${ }^{4}$, \\ Bruna Diirr ${ }^{1}$
}

${ }^{1}$ Graduate Program in Informatics - Federal University of the State of Rio de Janeiro (UNIRIO)

Postal Code 22290-250 - Rio de Janeiro - RJ - Brazil

${ }^{2}$ Graduate Program of Informatics - Federal University of Rio de Janeiro

Postal Code 21941-916 - Rio de Janeiro - RJ - Brazil

${ }^{3}$ Department of Industrial Engineering - Pontifical Catholic University of Rio de Janeiro (PUC-Rio)

Postal Code 22451-900 - Rio de Janeiro - RJ - Brazil

${ }^{4} \mathrm{SE7Ti}$ - Tecnologia e Inovação

Postal Code 22250-040 - Rio de Janeiro - RJ - Brazil

\{arthur.moreira, bruna.diirr\}euniriotec.br,

claudia.cappelli@gmail.com, fbaiaodinf.puc-rio.br, vanunes@gmail.com

\begin{abstract}
Democracy and citizen public participation, linked to the provision of government e-services (e-gov), have demanded transparency. How government works, data availability, quality, reliability, sources, and storage are issues that deserve attention and relate to the transparency concept. There are many interpretations of the term, and few are the researches proposing a concrete and common understanding and application. We propose OntoTrans, a Domain Ontology on Transparency that seeks to support the analysis, correlation, sharing, and reuse of such knowledge. An evaluation through example scenarios was performed, thus showing its feasibility and strengthening the research on the systematization of this applicability.
\end{abstract}

Resumo. A democracia e a participação pública dos cidadãos, ligadas à prestação de serviços eletrônicos de governo, têm exigido transparência. Como o governo funciona, disponibilidade, qualidade, confiabilidade, fontes $e$ armazenamento de dados são questões que merecem atenção e se relacionam ao conceito de transparência. Existem muitas interpretações do termo, e poucas são as pesquisas que propõem uma compreensão e aplicação concreta e comum. Propomos OntoTrans, uma Ontologia de Domínio sobre Transparência, que busca apoiar a análise, correlação, compartilhamento e reutilização de tais conhecimentos. Foi realizada uma avaliação por meio de cenários de exemplo, mostrando a sua viabilidade e fortalecendo a pesquisa sobre a sistematização dessa aplicabilidade.

\section{Introduction}

The actions that have been carried out in the most diverse countries for the development of Electronic Government programs (eGOV) have prioritized the use of information and 
communication technologies to democratize access to information. It aimed to broaden the debate and popular participation, as well as to improve the quality of public services and information provided. The eGOV of the Brazilian State follows a set of guidelines based on three fundamental ideas, one of them being citizen participation. However, it demands citizens to have total transparency of the government's information and processes, which takes us to a previous need to understand and to structure what is in fact the concept of transparency.

Transparency has been a desire for democratic societies for a long time. The right to be informed and to have access to public information has been a significant problem in modern societies. The demand for truth based on transparency has increased in the context of global change. The importance of openness in the flow of information is creating an open society in which the main idea is to establish a democratic society with engaged citizens able to understand and use the information that is accessible to them (Holzner and Holzner, 2006). However, it is not sufficient for an organization to wish to be transparent. Organizations need to know what transparency is and how they can apply this concept to their business.

Something transparent is something through which one can see, that is, something that allows or improves the view of a given object. In the context of organizations, transparency is something that allows or improves the vision of processes and information of an organization by giving an opportunity of knowledge about them, reduce the possibility of omission, allow for control over products and services provided, facilitate research, and increase trust between organizations and society. However, in order to invest in any initiative to meet the needs of making information and processes of an organization transparent, it is necessary to answer the question on how to make transparency explicit through processes and information.

Ontologies define knowledge structures and promote a shared understanding of a domain, task, or application (Chandrasekaran et al., 1998). They may take different forms, but typically involve several terms, a specification of their meanings, and how the terms are interrelated (Uschold and Jasper, 1999). The motivation behind the construction of an ontology on transparency was based on the advantages that this artifact can bring to this context, such as facilitating the information sharing on the domain of transparency, making more explicit assumptions, and assisting the knowledge and relations analysis in this domain.

This research work presents a Domain Ontology on Transparency and its encoding in OWL. OntoTrans seeks to support the analysis, sharing, and reuse of knowledge in this domain, as well as being a computational artifact capable of contributing to the understanding of the relations between other concepts that are related to transparency, and be processable by applications that manipulate this knowledge.

The paper is organized as follows: Section 2 describes the theoretical foundation on organizational transparency and ontologies; Section 3 presents the OntoTrans and the method used for its development; Section 4 discusses and evaluates the proposal; and, Section 5 concludes the paper and discusses further research perspectives. 


\section{Theoretical Foundation}

This section presents the main concepts related to this research: organizational transparency and why representing it as an ontology.

\subsection{Organizational Transparency}

Transparency is considered a concern for governments organizations. It represents the ideal condition for democratic societies, valuing access to information, participation, and accessibility in public relations and protection of citizens.

Transparency can be said as the availability of information through a variety of media about processes and information, allowing external actors to know about the actions and decisions taken in organizations. An aquarium is a good metaphor for transparency, and for years transparency has been often understood simply through these "light-shedding" metaphors (Albu, 2014), which allows "seeing through" and facilitating "clarity" and "understanding". Although, Christensen and Cheney (2013) state that transparency is equated mainly with giving information and accountability.

Cappelli (2009) adopted definitions such as the OECD (Economic Development Cooperation Organization), which says, "Transparency is a vital factor in strengthening relations between government and citizens". Therefore, Leite and Cappelli (2010) defined transparency as "The condition of the total opening of communication of an organization (company, institution, government) to the public, without any restriction of information", which we have been used due to their systematical property.

The transparency graph defined by Cappelli (2009) and Leite and Cappelli (2010) uses the NFR Framework (Chung et al., 2000) whose description language has syntax and semantics geared to Non-Functional Requirements (NFR) or Softgoals (clouds in Figure 1). Intrinsic to the language semantics is the notion that Softgoals does not have clear cut criteria for satisfaction, being a quality issue. It was Herbet Simon (1969) who coined the term "satisfice" to denote an outcome of a bounded rationality process that we believe was a proper realm to deal with this fuzzy concept, but trying, at the same time, to find ways of clarifying it from the perspective of quality relationships. As such, using the NFR language (Chung et al., 2000), we posit that transparency can be seen as a network of qualities that "help" transparency. Figure 1 presents this network, called the Softgoal Interdependence Graph (SIG).

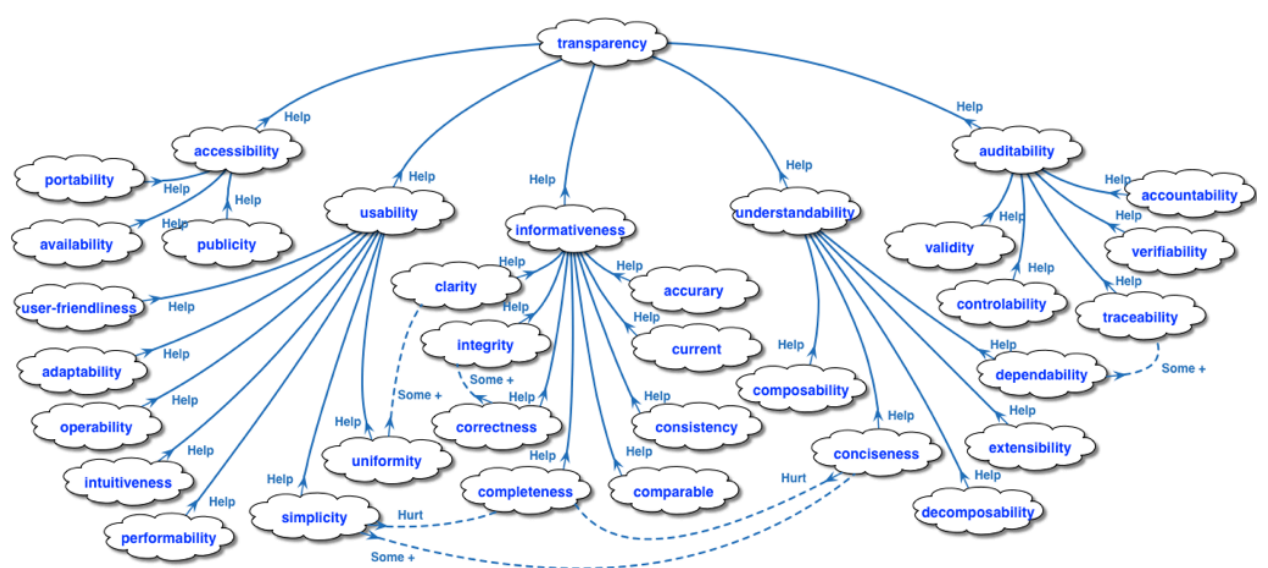

Figure 1. Transparency Graph (Cappelli, 2009; Leite and Cappelli, 2010). 


\subsection{Ontology}

Ontology is concerned with providing a system of categories that describes a particular view of the world. In Computer Science, they have been adopted and applied in different areas (Artificial Intelligence, Databases, Knowledge Representation, Natural Language Processing, Semantic Web, among others).

Gruber (1993) defined ontology as a formal, explicit specification of a shared conceptualization, one of the most quoted definitions of ontology in the semantic web literature. Ontologies define the structure of knowledge (Chandrasekaran et al., 1999) and promote a shared understanding of a domain, task, or application. They can be applied to aim at: (i) sharing information, (ii) reusing elements, (iii) making domain assumptions explicit, (iv) distinguishing domain knowledge from operational knowledge, and (v) analyzing domain knowledge (Guizzardi, 2005). Uschold and Jasper (1999) stated that an ontology typically comprises a vocabulary of terms, a specification of their meanings, and an indication of how the terms are interrelated.

Guarino (1998) defines a taxonomy of ontologies according to the degree of generality, defining four classes: (i) foundational ontologies, also named Top-Level or Upper ontologies, which comprise general, domain-specific concepts; (ii) domain ontologies, which describe the vocabulary of a specific domain of knowledge; (iii) task ontologies, describing events and activities and their inter-relation that take part in a generic task; and (iv) application ontologies, which specializes both domain and task ontologies for specific scenarios.

We propose OntoTrans as a domain ontology that aims to capture and conceptualize the required knowledge to understand what Transparency is and which are its essential characteristics, concepts, and relationships. OntoTrans is well-founded on UFO, a particular foundational ontology (Noy and McGuinness, 2001).

\subsection{The Unified Foundational Ontology (UFO) and OntoUML, its adjacent modeling language}

Foundational ontologies describe general concepts independent of a domain and precisely define meta-properties to make the semantics of each concept in the ontology explicit. Founding a domain ontology on foundational constructs enables highlighting tacit knowledge since it can express the underlying subtle ontological distinctions. The use of a foundational ontology as a basis during domain ontology specification also demonstrates that the rich choice of modeling primitives offered by the modeling language (representing these distinctions) as well as the methodological support of the formal meta-properties used, define them (e.g., rigidity, existential dependence) and forces the modeler to make the assumed ontological commitments explicit.

UFO is one example of a foundational ontology that has been developed based on several theories from Formal Ontology, Philosophical Logics, Philosophy of Language, Linguistics, and Cognitive Psychology (Guizzardi, 2005). It is composed of three main parts, namely UFO-A, UFO-B, and UFO-C. UFO-A is an ontology of endurants (objects). UFO-B is an ontology of perdurants (events and processes). UFO-C is an ontology of social entities (both endurants and perdurants) built on the top of UFO-A and UFO-B. As a structural conceptual model, OntoTrans concepts were founded in the constructs of UFO-A 
UFO-A elements are divided into Universals (types determining collections and concepts referring to things and beings) and Particulars (instances of such collections). In the Universal hierarchy, it is possible to determine classes (types) or collections of beings or material things that keep their identities even when submitted to changes. Such classes can be described through the following meta-properties: (i) Sortality, when objects keep its identity in every domain independently of changes it may suffer; (ii) Mixin, a property that can be applied to beings or things with different principles of identity categorizing sortals; (iii) Rigidity, a property that applies to the instances of a type in every given world where it can be found.

To develop OntoTrans, we used OntoUML as an ontologically well-founded modeling language represented as a UML profile (Guizzardi, 2005). OntoUML has been explicitly designed to have as modeling primitives those representing ontological distinctions prescribed by UFO. It has its real-world semantics defined in terms of several ontological theories, such as the theory of parts, wholes, types and instantiation, identity, dependencies, unity, etc. Therefore, OntoUML is a UML profile composed of a set of stereotypes, which represent the ontological categories of the UFO-A universals.

We used OLED (OntoUML Lightweight Editor https://code.google.com/p/ontouml-lightweight-editor) tool to create OntoTrans. It is a tool for development, evaluation, and implementation of domain ontologies in OntoUML. The tool provides a simple and integrated set of resources for ontology engineers such as syntactic checking, visual simulation, model checking, inference modeling, automatic detection of non-standard semantics and correction, validation of parthood relationships, and patterns of ontologies.

In order to instantiate OntoTrans, we used Protégé tool that implements OWL (Web Ontology Language), indicated by the W3C. This language describes classes, properties, and relationships between these conceptual objects to facilitate the interoperability of web content between machines. Protégé is a free open-source platform that provides a set of tools for building domain models and knowledge-based applications through ontologies. This set of tools includes a graphical interface for the definition of ontologies, deductive classifiers to validate consistent models, and infer information based on the analysis of an ontology protege.stanford.edu/about.php (protegewiki.stanford.edu/wiki/Main_Page).

\section{Related Work}

There are some works in the literature addressing transparency concerns in particular domains, such as e-Procurement (Muñoz-Soro et al., 2016) and customer complaints (Jarrat, 2009). Muñoz-Soro et al. (2016) proposed the PPROC ontology intending to publish, in a structured and standardized manner, public procurement information on public contracts, and their preparation. According to the authors, PPROC potentially improves efficiency in administration and facilitates access to public contracts, which undoubtedly contributes to increasing transparency in the particular e-Procurement domain. Jarrat (2009) proposed CContology, a customer complaint ontology that serves as the semantic backbone of an EU customer complaint portal. The CContology defines all the concepts related to a complaint in detail, to improve the effectiveness and transparency in e-business transactions. The closest work related to ours, however, is from Lourenco et al. (2016), which also addresses the lack of a conceptualization of 
transparency concerning public sector entities' use of resources. Interestingly, it also has been developed in the context of open government. However, it mainly addresses transparency for accountability concerns, and the ontology was developed from the analysis of newspaper news, following a "bottom-up" approach in which concepts emerge from the analyzed texts. We argue that this is complementary to the methodology we applied to develop OntoTrans that followed a "top-down" approach for concept definition and, therefore, is less subject to bias from the input data being considered.

\section{OntoTrans}

To develop OntoTrans, we adopted the ontology development methodology proposed by Ushold and King (1995) and extended by Uschold and M. Grüninger (1996). This methodology was chosen due to its simplicity and ease of use, its applicationindependence, the flexibility of its models, and the fact that it has been widely applied in several business domains (Gómez-Pérez et al., 2003). It is divided into four steps as follows. We used OntoUML for the construction using OLED Tool and generating an OWL code.

\subsection{Step 1: Identify Purpose}

The aim is that the reasons for the ontology construction and the relevant terms of the domain under study become clear.

Purpose of the construction: To establish relations between the transparency SIG elements proposed by Cappelli (2009), as well as to create a model that relates the transparency concepts, regardless of the context in which it is applied, which helps to make explicit the domain assumptions and that also can be consulted, shared and reused.

Relevant domain terms: Accessibility, Availability, Portability, Publicity, Auditability, Controllability, Accountability, Traceability, Validity, Verifiability, Understandability, Composability, Conciseness, Dependability, Extensibility, Decomposability, Informativeness, Accuracy, Current, Clarity, Comparable, Completeness, Correctness, Consistency, Integrity, Usability, Adaptability, Userfriendliness, Performability, Intuitiveness, Operability, Simplicity and Uniformity.

\subsection{Step 2: Building the Ontology}

Step 2 has three activities: Ontology Capture, Ontology Coding, and Integrating Existing Ontologies.

\section{Activity 2.1 - Ontology Capture}

The identification of the key concepts and the definition of the relations belonging to the domain were performed from the Transparency SIG proposed by Cappelli (2009).

Existing relationships in the domain: Hurt, Help, and Generate.

Key concepts of the domain: Accessibility, Auditability, Understandability, Informativeness, and Usability.

Uschold and Grüninger (1996) recommend the use of a middle-out approach to identify ontology concepts and their relationships. Since the concepts were previously 
defined by Cappelli (2009), we used a bottom-up approach to review the existing relationships. This approach was also used to propose new ones between the SIG concepts and during the construction of the textual definition of the ontology.

A new relationship called Need was proposed to describe that, in order to obtain the concept of the origin of the relationship, it is necessary to obtain the concept of destination. New relations of the types Help and Hurt were also proposed. However, since the transparency SIG was validated by a group of experts, an equally qualified group would have to validate any changes, which was not possible.

During OntoTrans construction, three approaches were considered. The first one was to create an ontology from this domain of research and the operationalization of its concepts in the business processes context, defined by Cappelli (2009). However, this approach was discarded since the ontology could be used only in the business processes context and not for information transparency, for example. The second one was the construction of a domain ontology and a task ontology to represent the operations suitable to the context in which it was decided to apply. It was also discarded because task ontologies are supposed to capture the knowledge of a problem's solution regardless of the domain, with solution details sufficient enough to reach the tasks' goal (Chandrasekaran et al., 1999; Gómez-Pérez et al., 2003). So, it would not be appropriate to create a task ontology to support OntoTrans in the business process domain.

The third approach was considered the most adequate, and the one that best conformed to the goals of this research work. In this approach, a "generic" domain ontology on transparency was constructed, which can be instantiated to help obtain transparency in several domains. To make it possible, a class Impact was added. It is generated by the leaf ontology concepts and, when instantiated, represents concrete forms of application of the transparency concepts that generated them. A partial view of the classes' textual description, according to the established pattern by the authors of the chosen method, is given below for the usability transparency key concept.

- Adaptability: Adaptability helps Usability and generates Impact.

- User-friendliness: User-Friendly helps Usability and generates Impact.

- Performability: Performability helps Usability and generates Impact.

- Intuitiveness: Intuitiveness helps Usability and generates Impact.

- Operability: Operability helps Usability and generates Impact.

- Simplicity: Simplicity helps Usability and Conciseness, hurts Completeness and generates Impact

- Uniformity: Uniformity helps Usability and Clarity and generates Impact.

\section{Activity 2.2 - Ontology Coding}

Figure 2 shows the OntoTrans model generated in the OLED tool. Several Impact classes were generated so that the diagram could remain readable; however, the Impact class is unique, and all the concepts (impact generators) are linked to it. OntoTrans was them transformed into an OWL model (including the SWRL structures for axiom formalization and ontology queries). We performed this using the OntoUML2OWL + SWRL (Barcelos et al., 2013) approach, available in the OLED tool. The expected 
result was a complete OWL code of the OntoUML ontology. However, the tool generated a file only with the OWL classes. This file, compatible with Protégé, was used to complete the construction of the ontology.

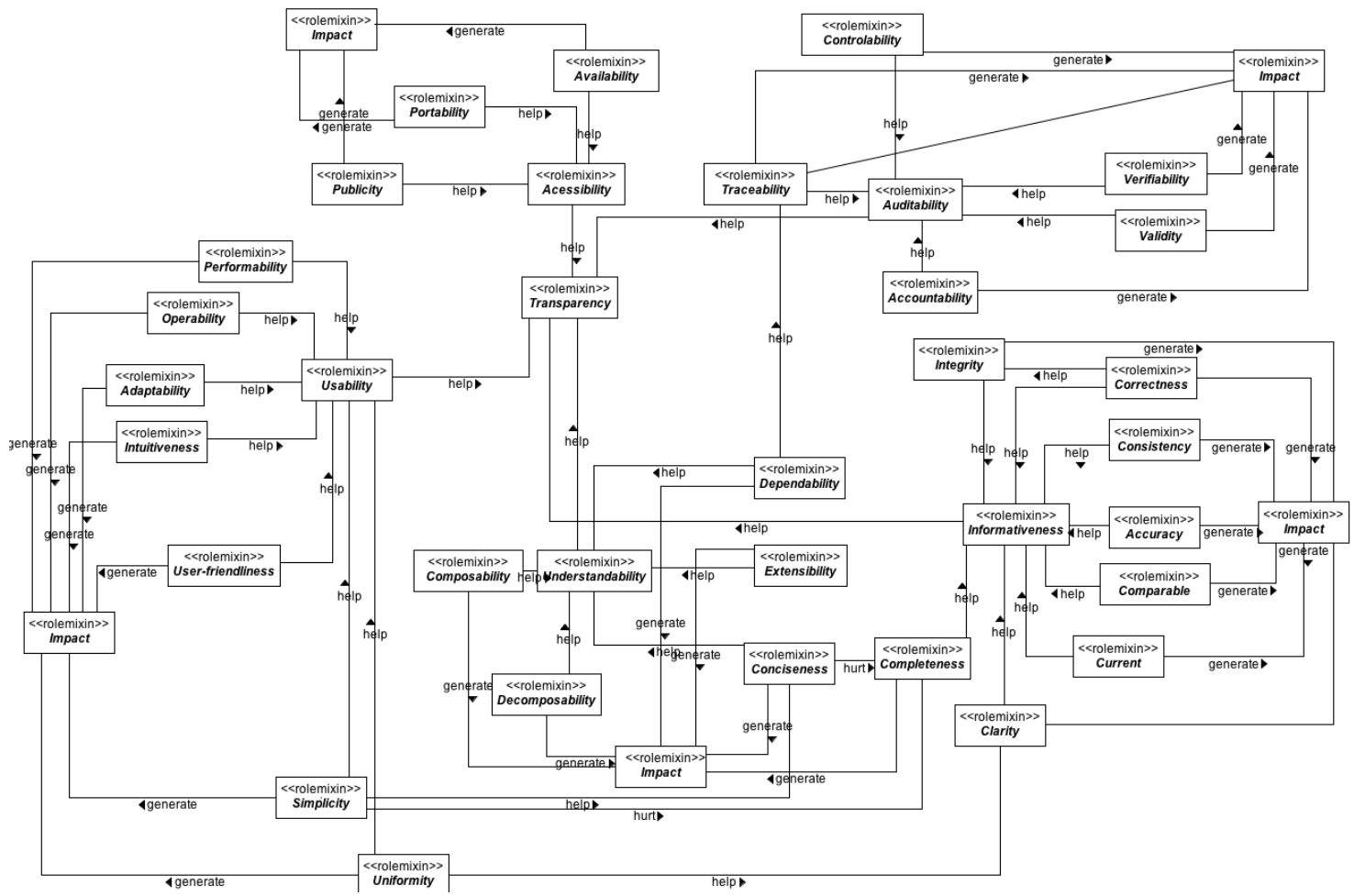

Figure 1. OntoTrans ontology model built on OLED tool using OntoUML language

Figure 3 shows the Object Properties, classes, and individuals generated in Protégé. Note that individuals were created for each ontology concept so that some queries could be realized. This ontology pre-instantiation also allows future OntoTrans users to apply it in their specific scenario, requiring only the instantiation of the impacts related to a particular domain.

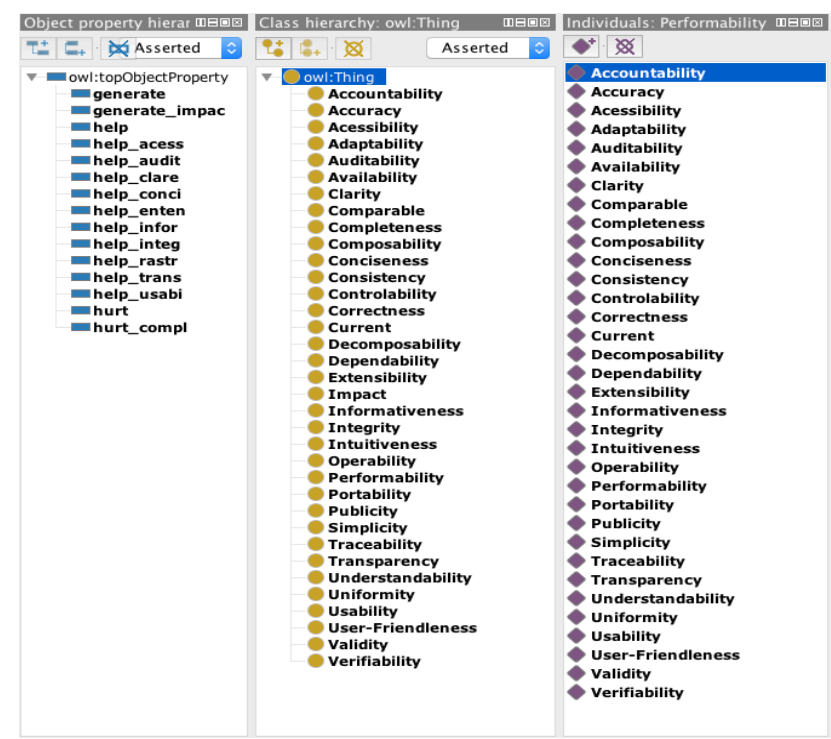

Figure 3. Protégé Object Properties, classes and Individuals of OntoTrans 


\section{Activity 2.2 - Integrating Existing Ontologies}

During the evaluation of the possibility of integrating existing ontologies, no ontology was integrated. Only UFO was used during the building Step.

\subsection{Step 3: Evaluation}

The OWL codification followed an existing approach (Barcelos et al., 2013) to avoid errors of manual conversion. Furthermore, an additional manual check was performed, searching each element of the conceptual model in the OWL ontology. No inconsistencies were found during this verification.

\subsection{Step 4: Documentation}

All steps performed were documented during the OntoTrans construction. Standards were used from the beginning to the end of its construction. All the terms used were defined, both generic and specific.

\section{Evaluation}

The Evaluation Step concerned with the correctness of OntoTrans to its conceptual model. Later, a capacity assessment of OntoTrans as a computational artifact capable of adding value when applying transparency in specific domains was performed. OntoTrans was instantiated in the Business Processes domain using SIG operations proposed by Cappelli (2009), where each one became an instance of the Impact class. The ontology instantiation allowed the realization of relevant queries and inferences. The queries should also be able to reaffirm the correctness of OntoTrans concerning its conceptual model, if they return the expected values. OntoTrans has been instantiated in the field of Business Processes. However, it can be instantiated for the understanding of goals and the realization of inferences related to transparency in any domain.

\subsection{Using OntoTrans}

DL Query, a native feature of Protégé, was used to perform queries. DLQuery uses a Manchester OWL syntax-based query language, which is a "user-friendly" syntax for OWL DL. The reasoner used was Protégé native HermiT2, in its version 1.3.8.413. Nine queries were performed, and the expected results, in Figures 4 to 6, were analyzed. Query 1: What are the concepts
that help usability in a business
process?

Expected result: Adaptability, User-friendliness, Performability, Intuitiveness, Operability, Simplicity, and Uniformity.

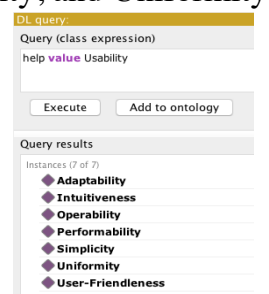

Query 2: What characteristics should a business process have in order to be auditable?

Expected result:

Controllability,

Accountability, Traceability, Validity, and Verifiability

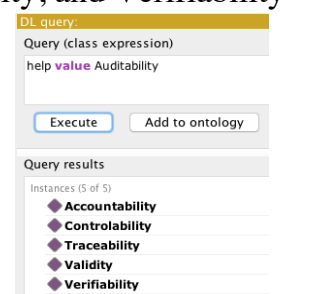

Query 3: What characteristics help to obtain the characteristics of understanding?

Expected result: Simplicity.

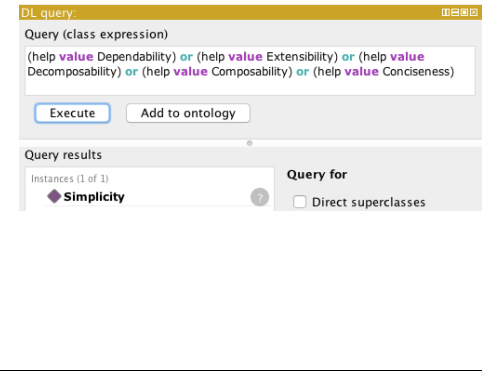

Figure 2. DL Queries 1, 2 and 3- code and result 
Query 4: What steps should we take to make this process accessible?

Expected result: The 12 actions displayed.

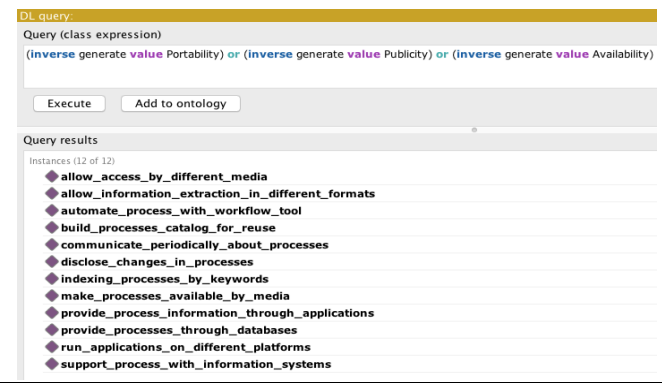

Query 5: What characteristics should a business process have in order to be accessible?

Expected result:

Accessibility, Portability, and publicity

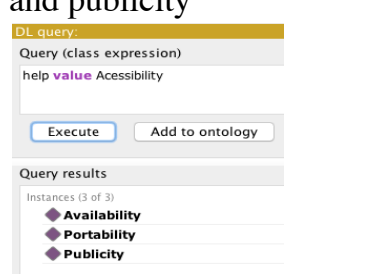

Query 6: What steps should we take to make a business process more detailed?

Expected result: The two actions displayed.

Query (class expres sion) Query (class (aplession) (inverse generate value Extensibility) Execute Add to ontology Query results decompose_informatio

Figure 3. DL Queries 4, 5 and 6 - code and result

\begin{tabular}{|c|c|c|}
\hline $\begin{array}{l}\text { Query 7: What characteristics of } \\
\text { Understandability is helped } \\
\text { Simplicity? }\end{array}$ & $\begin{array}{l}\text { Query 8: What steps should we } \\
\text { take to make a business process } \\
\text { clearer? }\end{array}$ & $\begin{array}{l}\text { Query 9: Which concepts } \\
\text { can undermine the } \\
\text { completeness of a business }\end{array}$ \\
\hline Expected result: Conciseness. & $\begin{array}{l}\text { Expected result: The seven } \\
\text { actions displayed. }\end{array}$ & $\begin{array}{l}\text { process? } \\
\text { Expected result: }\end{array}$ \\
\hline $\begin{array}{l}\text { Query (class expression) } \\
\text { (inverse help value Simplicty) and (help value Understandability) }\end{array}$ & $\begin{array}{l}\text { Dh quenv } \\
\text { Querv (class expression) } \\
\text { (inverse generate value Clarity) }\end{array}$ & $\begin{array}{l}\text { Conciseness and } \\
\text { Simplicity. }\end{array}$ \\
\hline Execute Add to ontology & Execute Add to ontolagy & Query (class expression) \\
\hline Query results & Querv results & (hurt value Completeness) \\
\hline $\begin{array}{ll}\mid \text { Instances }(1 \text { of } 1) \\
\forall \text { conciseness } & \begin{array}{l}\text { Query for } \\
\text { Direct superclasses }\end{array}\end{array}$ & 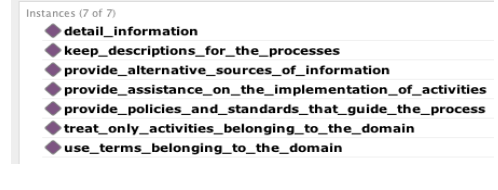 & $\begin{array}{l}\text { Execute Add to ontology } \\
\text { Query results } \\
\text { Instances (2 of } 2) \\
\text { conciseness } \\
\text { Simplicity }\end{array}$ \\
\hline
\end{tabular}

Figure 4. DL Queries 7, 8 and 9 - code and result

\subsection{Final Considerations}

In the evaluation of the ontology, it was possible to extract relevant knowledge about transparency that was encoded within the ontology and would not easily be accessed through its structure. The results of the queries provided a strong indication of the correctness of the OWL ontology concerning its conceptual specification, corroborating with the statement made in the OntoTrans section.

The queries indicate that OntoTrans is capable of assisting the sharing of the transparency domain information and assisting the analysis of this domain, making its assumptions more explicit. Moreover, the built ontology can provide these characteristics in specific domains, in this case, being necessary its instantiation for the desired domain, as performed for the business process domain in this evaluation.

The evaluation results also indicate that OntoTrans can assist in automating the application of transparency. The results of queries combined with an application could, for example, automate the insertion of transparency activities into an organization's process models. Besides that, results also indicate that the built ontology is a computational artifact capable of adding value in scenarios of application of transparency in the most varied domains since it is "generic", and can be instantiated in the domain of choice of the user. 
From the citizen's point of view, having a defined ontology on transparency is also very important. The fact that there is a consensus for all government agencies about this concept makes everyone present and evaluate transparency about information under their responsibility with the same characteristics. Another benefit is also the understanding of the presented contents by the citizen that can use the ontology as a source of consultation

\section{Conclusions}

Being transparent is a necessary characteristic of many public, private, and nonprofit organizations. However, there are still many interpretations of this term. Cappelli (2009) organized all this knowledge through an NFR Framework (Chung et al., 2000), allowing the definition of concepts that have contribution relationships among them.

From the SIG proposed by Cappelli (2009), the characteristics of transparency and the existing relationships among them were identified and structured in order to characterize OntoTrans. Such creation made use of languages like OntoUML and OWL, besides tools like OLED and Protégé for the generation of a Generic Transparency Ontology that can be instantiated in several domains. In order to instantiate Ontology in one domain, it is necessary to discover the generated impacts (actions that contribute to obtaining) for each leaf concept of the ontology in the desired domain.

In order to test the built-in Ontology and evaluate the benefits obtained by constructing it, an OntoTrans instance for the Business Processes domain was built on Protégé. For the Ontology test, the DLQuery tool was used to perform queries. The queries returned the expected results. The built instance demonstrated that Ontology is an effective computational tool for the understanding of how to apply transparency in the Business Process domain providing information for the user to make inferences. The OWL technology makes it compatible with standards of the Semantic Web and with other standards used by the $\mathrm{W} 3 \mathrm{C}$, facilitating their sharing and reuse.

This work was limited to the concepts that help to obtain transparency. Concepts that hinder the achievement of transparency were not added. Besides that, the tests and queries performed on OntoTrans were limited to just one knowledge domain.

Future work may include the ontology instantiation in an e-Gov, along with the evaluation of experts in the selected fields and experts in transparency to point out the benefits generated by OntoTrans in understanding the transparency domain and in understanding and facilitating the application of transparency in a specific domain. Another future application is the use of OntoTrans to implement more transparent information systems.

\section{References}

Albu, O.B. (2014). "Transparency in Organizing: A Performative Approach". PhD Thesis. Copenhagen Business School.

Barcelos, P.P.F., V.A. Santos, F.B. Silva, M.F. Monteiro and A.S. Garcia (2013). "An Automated Transformation, from OntoUML to OWL and SWRL". In: Ontobras.

Cappelli, C. (2009). "An approach for Business Processes Transparency Using Aspects". PhD Thesis, PUC-Rio (in Portuguese). 
Chandrasekaran, B., J.R. Josephson and R. Benjamins (1998). "The Ontology of Tasks and Methods". In: Knowledge Acquisition Modeling and Mngt. Workshop, Banff.

Chandrasekaran, B., J.R. Josephson and R. Benjamins (1999). "What are Ontologies and What Do We Need Then". IEEE Intelligent Systems 14(1), 20-26.

Christensen, T. and G. Cheney (2013). "Peering into Transparency". In: Third Global Conference on Transparency Research, Paris. URL: http://campus.hec.fr/globaltransparency/?page_id=281.

Chung, L., B. Nixon, E. Yu and J. Mylopoulos (2000). Non-Functional Requirements in Software Engineering. Massachusetts: Kluwer Academic Publishers.

Gómez-Pérez, A., M. Fernandéz-López and O. Corcho (2003). "Methodologies and methods for Building Ontologies". Advanced Information and Knowledge Processing, 107-197.

Gruber, T.R. (1993). "A Translation approach to portable ontologies". Knowledge Acquisition 5(2), 199-220.

Guarino, N. (1998). "Formal Ontology and Information Systems". In: International Conference on Formal Ontology in Information Systems, Trento.

Guizzardi, G. (2005). Ontological Foundations for Structural Conceptual Models. The Netherlands: Universal Press.

Holzner, B. and L. Holzner (2006). Transparency in Global Change: The Vanguard of the Open Society. University of Pittsburgh Press.

Jarrat, M. (2009). Towards effectiveness and transparency in e-business transactions: an ontology for customer complaint management. In Semantic Web for Business: Cases and Applications (pp. 127-149). IGI Global.

Leite, J.C.S. and C. Cappelli (2010). "Software Transparency". Business \& Information Systems Engineering 2(3), 127-139.

Muñoz-Soro, J. F., Esteban, G., Corcho, O., \& Serón, F. (2016). PPROC, an ontology for transparency in public procurement. Semantic Web, 7(3), 295-309.

Noy, N.F. and D.L. McGuinness (2001). "Ontology Development 101: A Guide to Creating Yout First Ontology”. Technical Report KSL-01-05, Stanford University.

Simon, H. (1969). The Sciences of the Artificial. MIT Press.

Sowa, J.F. (2004). "Principles of Ontology". URL: www.-ksi.stanford.edu/onto$\mathrm{std} /$ mailarchive/0136.html.

Uschold, M. and M. Grüninger (1996). "Ontologies: Principles, Methods and Applications". Knowledge Engineering Review 11 (2), 93-155.

Uschold, M. and R. Jasper (1999). “A Framework for Understanding and Classifying Ontology Applications". In: Workshop on Ontology and Problem Solving Methods: Lessons Learned and Future Trends, Stockholm.

Uschold, M. and M. King (1995). "Towards a Methodology for Building Ontologies". In: Workshop on Basic Ontological Issues in Knowledge Sharing, Montreal. 